\title{
An Analysis \& Survey on Quality of Service Improvement in MANET, VANET and WSN
}

\author{
Amit Yogi ${ }^{1}$, Jayesh Surana ${ }^{2}$ \\ ${ }^{1}$ Pursuing M. Tech, SVITS, Indore, India \\ ${ }^{2}$ Assistant Professor, SVITS, Indore, India
}

\begin{abstract}
An Ad-hoc network has a Provision of Quality of Service (QoS) to support many applications like digital and multimedia applications. However, one of the challenging tasks in Ad-hoc Network (MANET \&VANET) is Quality of Service (QoS) which is determined by numerous parameters such as bandwidth and delay constraints, varying channel conditions, power limitations, node mobility, dynamic topology, packet delivery ratio, end-to-end delay and connection duration. With the increasing demand for real time applications in the Wireless Senor Network (WSN), real time critical events anticipate an efficient quality-of-service (QoS) based routing for data delivery from the network infrastructure. Designing such QoS based agent routing protocol to meet the reliability and delay guarantee of critical events while preserving the energy efficiency is a challenging task. This paper surveying about (QoS) based agent routing algorithms in MANET, WSN and VANET.
\end{abstract}

Keywords: MANET, QOS, VANET, WSN,

\section{Introduction}

Mobile ad hoc network (MANET) is a decentralized, selforganizing wireless network without any fixed infrastructure. Each node in a MANET behaves not only as a host, but also as a router [1]. Mobile multimedia ad hoc networks have created great demand in the services for the mobile users that require stringent Quality of Service (QoS). However, there are several problems and issues which have to consider for QoS support in MANETs including signaling, medium access control, security, reservation, and routing. Routing is considered as one of the most important aspect of MANET due to the dynamic topology. Eventhough wireless ad hoc networking researchers have addressed the routing problem since a decade; they have still not yet come up with a robust and efficient routing scheme. Thus, we have scope to develop efficient routing protocols for multimedia applications to decrease routing related overheads and find QoS routes with better packet delivery ratio, higher throughput and lower delays.

Table-driven (proactive), on-demand (reactive) and hybrid routing protocols are three main categories of routing protocols for ad hoc wireless networks. Table driven routing algorithms include Destination Sequenced Distance Vector (DSDV), Clustered Gateway Switch Routing (CGSR) and Wireless Routing Protocol (WRP). On demand routing algorithms include past decades, it has received tremendous attention from both academia and industry all over the world. A WSN typically consists of a large number of lowcost, low-power, and multifunctional wireless sensor nodes, with sensing, wireless communications and computation capabilities [4,5]. These sensor nodes communicate over short distance via a wireless medium and collaborate to accomplish a common task, for example, environment monitoring, military surveillance, and industrial process control [6]. The basic philosophy behind WSNs is that, while the capability of each individual sensor node is limited, the aggregate power of the entire network is sufficient for the required mission.
Quality of Service (QoS) means that the network should provide some kind of guarantee or assurance about the level or grade of service provided to an application. The actual form of QoS and the QoS parameter to be considered depends upon specific requirements of an application. For example, an application that is delay sensitive may require the $\mathrm{QoS}$ in terms of delay guarantees. Some applications may require that the packets should flow at certain minimum bandwidth. In that case, the bandwidth will be a QoS parameter [7]. Certain application may require a guarantee that the packets are delivered from a given source to destination reliably, then, reliability will be a parameter for QoS.

\section{Characteristics of MANET and WSN}

MANET: Dynamic Source Routing (DSR), On-Demand Distance Vector Routing (AODV), Temporally Ordered Routing Algorithm (TORA) and Zone Routing Protocol (ZRP). Hybrid routing algorithms aim to use advantages of table driven and on demand algorithms and minimize their disadvantages. Ant colony Mobile agent based algorithms are a special category of algorithms (proactive, reactive and hybrid) that provide features such as adaptively and robustness which essentially deal with the challenges of the MANETS.

VANET: A Vehicular Ad hoc Network (VANET) is a form of wireless ad hoc network to provide communications among vehicles and nearby roadside equipments. It is emerging as a new technology to integrate the capabilities of new generation wireless networking to vehicles. The major purpose of VANET is to provide (2) ubiquitous connectivity while on the road to mobile users, who are otherwise connected to the outside world through other networks at home or at the work place, and efficient vehicle-to-vehicle communications that enable the Intelligent

WSN: Wireless sensor network (WSN) is widely considered as one of the most important technologies for the twenty- 


\section{International Journal of Science and Research (IJSR)}

ISSN (Online): 2319-7064

Index Copernicus Value (2013): 6.14 | Impact Factor (2014): 5.611

first century [3].As compared to the traditional wireless communication networks such as mobile ad hoc network (MANET) and cellular systems, wireless sensor networks have some different properties when compared with MANET.

ROUTING ALGORITHMS FOR MANET VANET AND WSN: The Ad hoc On demand Distance Vector (AODV) algorithm is a demand based protocol. Using distance vectors, each node stores available routes for known destinations. AODV floods a "Route Request"e (RREQ) message to its neighbors to discover new routes. Each Route request RREQ message propagates through the network until it reaches the destination or a node that has a fresh route to the destination. AODV uses only the shortest path to transfer data and does not require nodes to store routes to destinations they do not communicate with.

Dynamic Source Routing (DSR) is also based and, like AODV, uses only the shortest discovered path. When a node wants to send a message, it broadcasts a route request message to its neighbors which add their own addresses and rebroadcast the message until it reaches the destination which replies using the discovered path. When a failed link is detected, a message is sent to the source and the route is discarded. DSR then rebroadcasts to discover a new route. DSR supports both bidirectional and unidirectional links.

The Cluster Based Routing Protocol (CBRP) is another demand based protocol that divides the MANET nodes into a set of clusters. One host is elected as each cluster's head "which maintains the clusters membership information. The need for inter cluster routing is discovered using the membership information and is performed by the cluster heads. CBRP is useful for large scale MANETs since the clustering reduces the number of routing messages needed.

Optimized Link State Routing (OLSR) [8] is a proactive protocol. Each node selects a set of neighbors as multipoint relays (MPRs) which periodically announce their existence to the network. MPRs are used to find routes in the network. Unlike CBRP, OLSR selects MPRs dynamically.

\section{Topology Broadcast using Reverse Path Forwarding (TBRPF)}

[9] is another proactive protocol that uses shortest paths. Each node maintains a ,source tree ${ }^{e e}$ of shortest paths to its reachable nodes and announces a part of its source tree to its neighbors using a combination of periodic and triggered updates. To decide which part of the source tree to send, a node $\mathrm{i}$ computes its reportable node set ${ }^{\mathrm{ee}}(\mathrm{RN})$. Node i puts node $\mathrm{u}$ in $\mathrm{RN}$ if it determines that some neighbor $\mathrm{j}$ may select node $i$ to be the next hop on a shortest path from $j$ to u.

The Fisheye State Routing protocol (FSR) [10] is also a proactive protocol. A "center" node in the MANET stores link state information for all nodes. This node periodically sends the information for all nodes to its r-hop neighbors $(r$ $=1,2, .$.$) . This is done at different frequencies based on the$ value of $r$. In FSR, nodes can obtain the entire network topology and therefore compute efficient routes.

\section{Agent and Algorithms Used in MANET}

Agent based QoS routing: The scheme uses a mobile agent to perform this operation. Every node in a network comprises of an agent platform. We assume that every node in a network maintains an agency for QoS routing.[14].

User Monitoring Agent (UMA):It is a static agent which is present in all nodes that monitors the request generated at the node. If demand generated, this agent triggers the DSR Agent (DSRA) and QoS Agent (QoSA) and also this agent computes the residual bandwidth, delays, jitters and packet losses for each link and updates the QoS status profile at regular intervals. All the parameters are computed within a given continuous time window this database sends to the QoSA for future computation.

Neuro-Fuzzy Agent (NFA): It is a static agent, when there is demand at the node, automatically this agent triggers and start straining of data sets defined for multimedia communication till acceptable error limit.

DSR Agent (DSRA): It is a Static agent. When the request from the UMA, it starts discovery of multipath from source to destination and selects zDMR with stable paths. After finding it requests to QoS agent for QoS selection of each path.

QoS Agent (QoSA): It is also a static agent. This receives two requests, first from UMA that demands to compute requested QoS for particular application using optimized membership values and second request from DSRA to check QoS at each node according to requested QoS. After this, this provides QoS paths for communication.

Route Maintenance Agent (RMA): It is a mobile agent, which migrates along the path which is communicating. If any link failure due to node mobility or failure of node, this agent finds alternate path to destination from disconnected link.

\section{Algorithm used in MANET}

step 1: Optimize the Membership functions by training of input data set vector using error back propagation algorithm.

step 2: If any demands from the user in the network, then, Start multipath route discovery else

Update the network and go to step 2.

step 3: Get the QoS satisfied paths among stable paths using trained data set vector and fuzzy logic.

step 4: If QoS paths are present, If only one QoS path, then, start transmission of packets through it.

Else

Select one best QoS path and start the transmission of packets through it. Else update the network and go to step 2.

step 5: If any route failure, then, start route discovery from disconnected node and go to step 3. Else update the 


\section{International Journal of Science and Research (IJSR)}

network and go to step 2.

End.

\section{Agent and Algorithms Used in VANET}

In Vehicular Agent (IVA): IVA is static agent resides in vehicle which communicates with the DFA to acquire/spread the relevant information. IVA collects the status (moving or stationary) and location information of vehicle from sensors equipped in a vehicle.

Observant Agent (OA): OA is a mobile agent that travels around the network by creating its clones to propagate the decisive information during the critical situations. Examples of critical situation are accident, traffic jam, bad weather conditions, tracing a vehicle involved in crime or traffic rule violation etc. It also informs IVA and updates the vehicle database. OA is sent by DFAs to the vehicles moving in the network.

Information Finding Agent (IFA): IFA travels in the network to search for the requisite information as desired by vehicle user. IFA is sent by the DFA in the network on the request issued by user or DFA itself to get traffic information. [15]

\section{Routing Algorithms for WSN}

\section{Flat Routing}

In flat networks, each node typically plays the same role and sensor nodes collaborate together to perform the sensing task. Due to the large number of such nodes, it is not feasible to assign a global identifier to each node.

\section{Sensor Protocols for Information via Negotiation (SPIN)}

SPIN disseminate all the information at each node to every node in the network assuming that all nodes in the network are potential base-stations [17].

\section{Directed Diffusion}

As a data-centric protocol, applications in sensors label the data using attribute-value pairs. A node that demands the data generates a request where an interest is specified according to the attribute-value based scheme defined by the application. The sink usually injects an interest in the network for each application task [18]. The nodes update an internal interest cache with the interest messages received. The nodes also keep a data cache where the recent data messages are stored. This structure helps on determining the data rate. On receiving this message, the nodes establish a reply link to the originator of the interest. This link is called gradient and it is characterized by the data rate, duration and expiration time. Additionally, the node activates its sensors to collect the intended data. The reception of an interest message makes the node establish multiple gradients (or first hop in a route) to the sink. In order to identify the optimum gradient, positive and negative reinforcements are used. There algorithm works with two types of gradients: exploratory and data gradients. Exploratory gradients are intended for route setup and repair whereas data gradients are used for sending real data.

\section{Rumor Routing}

The Rumor Routing protocol improves a nodes ability to transmit queries and event information throughout a wireless sensor network. The most expedient way to guarantee every query is successful is to flood the WSN with both query and event information [19] [20]. Each node within a WSN with Rumor Routing initializes using an active broadcast to locate neighboring nodes. These neighbors are added to a list within the nodes memory, which is maintained through subsequent active broadcasts, or by passively listening to other nodes" broadcasts. Additionally, each node maintains an event table containing forwarding information for each event it has been informed of. If a node witnesses an event, it adds it to its event table and generates an agent. The agent traverses the network, "informing" other nodes of events It has witnessed. The agent uses a straightening algorithm to maintain a straight path, thereby transmitting information as far across the network as possible. The agent contains a list of witnessed events as well as the number of hops to each event. When received by a node, the agent synchronizes its list with the nodes list so both of their tables contain routes to every event. In addition, since agents are broadcast in the WSN, every neighboring node within receiving distance of the agent receives the updated information and updates their event tables as well. This behavior continues until the agent's lifetime expires. To receive event information, a node within the WSN generates a query. The query is sent in a random direction to a neighboring node. That node, if aware of a route to the event, forwards the query accordingly. Otherwise, it forwards the query in a random direction to one of its neighboring nodes. The query uses the same algorithm as the agent to determine the direction to send the query, thus avoiding the same nodes. Should a node within the network fail, however, it is possible the query could be caught in a loop. To avoid this, each query is assigned limited lifetime, as well as a random identification number. If a query arrives at a node which has already forwarded it, the node instead sends the query to a random neighbor, thus breaking the loop. This process continues until the query has reached a node that has information about the event, or until the query's lifetime expires. If the originating node of a query determines it did not reach the event, it can retransmit the query, quit the query, or flood the network with the query.

\section{Conclusion}

QoS aware routing in MANET, VANET and WSN is a challenging task. Many research works have been carried out in this area. In this paper, comprehensive survey of QoS based Agent routing algorithm is proposed which supports real time and multimedia applications. These algorithms are more adaptive and energy efficient which takes node's remaining energy as well as drain rate (i.e. energy dissipation rate) as QoS parameter. It selects the node which has sufficient resource to satisfy the QoS constraints. In high mobility cases it is very efficient in terms of quick route maintenance. These algorithms are takes care of end to end delay, available bandwidth, and hop count as QoS parameter which increases network throughput. This paper clearly 


\section{International Journal of Science and Research (IJSR) \\ ISSN (Online): 2319-7064}

Index Copernicus Value (2013): 6.14 | Impact Factor (2014): 5.611

explains the characteristics of MANET, VANET and WSN and also analysis the QoS based agent routing algorithms in MANET, VANET and WSN

\section{References}

[1] D. O. Schmidt and R. Trentin, "MANETs Routing Protocols Evaluation in a Scenario with High Mobility MANET Routing Protocols Performance and Behavior", Proceeding of IEEE Network Operations and Management Symposium, (2008), pp. 7-11.

[2] Naishadh K. Dave and Vanaraj B. Vaghela "Vehicular Traffic Control: A Ubiquitous Computing Approach", IC3 2009, CCIS 40, pp. 336-348, 2009.

[3] "21 ideas for the 21 st century", Business Week, Aug. 301999 , pp. 78-167.

[4] S.K. Singh, M.P. Singh, and D.K. Singh, "A survey of Energy-Efficient Hierarchical Cluster-based Routing in Wireless Sensor Networks", International Journal of Advanced Networking and Application (IJANA), Sept.-Oct. 2010, vol. 02, issue 02, pp. 570-580.

[5] S.K. Singh, M.P. Singh, and D.K. Singh, "Energyefficient Homogeneous Clustering Algorithm for Wireless Sensor Network", International Journal of Wireless \& Mobile Networks (IJWMN), Aug. 2010, vol. 2, no. 3, pp. 49-61.

[6] Jun Zheng and Abbas Jamalipour, "Wireless Sensor Networks: A Networking Perspective", a book published by A John \& Sons, Inc, and IEEEE, 2009.

[7] Hanzo, L. and Tafazolli, R. (2007) „A survey of QoS routing solutions for mobile ad hoc networksee, IEEE Communications Surveys, Vol. 9, No. 2, pp.50-70

[8] Jacquet.P, Muhletaler.P, Qayyum.A, Clausen.T, and Viennot.L : "Optimized LinkState Routing Protocol". In Proceedings of IEEE International Multi Topic Conference. Lahore, Pakistan, December 2001.

[9] Ogier.R, Templin.F, Bellur.B and Lewis.M: "Topology Broadcast Based on ReversePathForwarding (TBRPF)". Internet draft, March, 2002.

[10]Pei.G, Gerla.M, and Chen.T: "Fisheye State Routing in Mobile Ad Hoc Networks" in Proceedings of the Workshop on Wireless Networks and Mobile Computing. Taipei, Taiwan, April 2000, pp. D71D78.

[11]Colorni, A., Dorigo M. \&ManiezzoV., "The Ant System: Optimization by a colony of co- operating agents", IEEE Transactions on Systems, Man, and Cybernetics-part B, Vol. 26, No.1, pp.1-13, 1996. http://dx.doi.org/10.1109/3477.484436.

[12] Gianni Di Caro and Marco Dorigo, "AntNet: distributed stigmergetic control for communication networks", Journal of Artificial Intelligence Research, vol. 9, pp. 317-365, 1998.

[13] Gianni Di Caro, Frederick Ducatelle and Luca Maria Gambardella, "AntHocNet: an adaptive nature-inspired algorithm for routing in mobile ad hoc networks", European Transactions on Telecommunication, Volume 16, pp: 443-455, 2005. http://dx.doi.org/10.1002/ett.1062

[14] Manvi.S.S and Telsang.V "An Agent based approach to QOS Routing in Mobile ADHOC Network". 2004 International Conference on Signal Processing \& Communications (SPCOM):8690.

[15]Brijesh Bhatt VasundharaUchhula. "Comparison of different Ant Colony Based Routing Algorithms".International Journal of Computer Applications (IJCA).Vol. 2.Pp. 97-101, 2010. http://dx.doi.org/10.5120/1040-65.

[16]Neeran M. Karnik and Anand R. Tripathi "DesignIssues in Mobile-Agent Programming Systems", IEEE Concurrency, July-September, 1998 pp 52-61.

[17]Perrig, R. Szewzyk, J.D. Tygar, V. Wen, and D. E. Culler, "SPINS: security protocols for sensor networks". Wireless Networks Volume: 8, pp. 521-534, 2000.

[18] Intanagonwiwat, R. Govindan, and D. Estrin, "Directed diffusion: a scalable and robust communication paradigm for sensor networks," Proceedings of ACM MobiCom '00, Boston, MA,2000, pp. 56-67.

[19]D. Braginsky and D. Estrin, "Rumor routing algorithm for sensor networks," in Proceedings of the1st ACM International Workshop on Wireless Sensor Networks and Applications, pp. 22-31, 2002.

[20]Braginsky and D. Estrin, "Rumor Routing Algorithm for Sensor Networks," in the Proceedings of the First Workshop on Sensor Networks and Applications(WSNA), Atlanta, GA, October 2002. 